\title{
Générer des données d'essais randomisés pour optimiser le traitement à l'ère de la pandémie de COVID-19
}

\author{
Matthew P. Cheng MDCM, Todd C. Lee MD MPH, Darrell H.S. Tan MD PhD, Srinivas Murthy MDCM MHSC
}

- Citation : CMAJ 2020 April 14;192:E405-7. doi : 10.1503/cmaj.200438-f; diffusion hâtive le 31 mars 2020

Voir la version anglaise de l'article ici : www.cmaj.ca/lookup/doi/10.1503/cmaj.200438

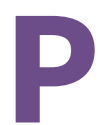

endant que des stratégies de prévention énergiques sont appliquées pour ralentir la propagation du coronavirus du syndrome respiratoire aigu sévère 2 (SRAS-CoV-2), nous devons aussi optimiser les résultats cliniques chez les personnes infectées et prévenir la transmission à leurs proches. À mesure que le nombre de patients gravement malades de la maladie à coronavirus 2019 (COVID-19) continue d'augmenter, il faut dans les meilleurs délais en apprendre le plus possible sur cette maladie afin d'orienter les soins. Heureusement, dans un effort concerté, les scientifiques canadiens en recherche clinique se sont déjà mobilisés pour mettre sur pied des essais randomisés et contrôlés $(E R C)$ sur des modalités thérapeutiques, prophylactiques et vaccinales. En collaboration avec des collègues du monde entier, nous cherchons à déterminer pourquoi et comment les chercheurs canadiens se positionnent pour générer des données fiables et améliorer les résultats chez les patients, ici et ailleurs dans le monde.

Face à une maladie inconnue et terrifiante comme la COVID19, et à des taux de mortalité potentiellement élevés et préoccupants, certains médecins et patients seront attirés par des traitements non éprouvés en se fiant à des spéculations, des données in vitro, des modèles animaux, des anecdotes cliniques, des études d'observation parasitées par des biais de confusion quant à la gravité des cas et des essais cliniques non contrôlés ou dotés d'une puissance statistique insuffisante qui pourraient ultérieurement se révéler erronés ${ }^{1-3}$. Par exemple, durant l'épidémie de grippe H1N1 (virus influenza de type A) en 2009, les pays se sont constitué des réserves d'oseltamivir à prix d'or et les ont beaucoup utilisées sur la base de données de qualité sousoptimale qui montraient un bénéfice potentiel chez les patients hospitalisés ${ }^{4}$. Encore aujourd'hui, étant donné qu'il n'y a pas eu d'ERC, on ne peut conclure hors de tout doute à l'efficacité de l'oseltamivir pour la prévention ou le traitement des complications de la grippe chez les patients hospitalisés.

En ce qui concerne la pandémie de COVID-19, des médicaments expérimentaux qui ne sont approuvés actuellement pour aucune indication dans aucun pays ont déjà été largement

\section{POINTS CLÉS}

- Face à une maladie inconnue et terrifiante comme la COVID-19 et à des taux de mortalité potentiellement élevés et préoccupants, certains médecins et patients seront attirés par des traitements non éprouvés.

- Nous devons éviter les soins arbitraires et aléatoires et veiller à ce que les patients aient plutôt accès à des soins randomisés, par assignation aux traitements disponibles les plus prometteurs ou aux groupes témoins, comme le suggère l'Organisation mondiale de la santé, de manière à générer le plus rapidement possible des données sur les traitements les plus sécuritaires et efficaces.

- L'Organisation mondiale de la santé a priorisé plusieurs médicaments à explorer dans le contexte de la COVID-19, dont le lopinavir-ritonavir, le remdésivir et l'hydroxychloroquine.

- L'infrastructure canadienne en recherche clinique subit une mise à l'échelle rapide pour relever le défi et les instances de réglementation agissent avec célérité pour faciliter le démarrage des premiers essais canadiens sur la COVID-19 qui s'inscrivent dans l'effort de recherche mondial pour étudier des stratégies thérapeutiques et prophylactiques.

utilisés en dehors de protocoles d'essais cliniques 5 . Mais un argument éthique et clinique de poids milite pour que ces soins arbitraires et aléatoires soient remplacés par des soins "randomisés ", c'est-à-dire, dans le cadre de protocoles établis où les patients sont d'office assignés aux options disponibles les plus prometteuses ou aux groupes témoins, de manière à générer le plus rapidement possible des données sur les traitements les plus sécuritaires et efficaces. Cela signifie que même si les premiers patients risquent de recevoir des traitements sur une base spéculative, les patients subséquents pourront recevoir des traitements plus sécuritaires, fondés sur des données probantes.

À partir de données in vitro, de données d'innocuité publiées et de leur plausibilité biologique, l'Organisation mondiale de la santé (OMS) a priorisé plusieurs médicaments à explorer pour la 
COVID-19 et en a recommandé l'évaluation dans le cadre d'essais cliniques (www.who.int/blueprint/priority-diseases/key-action/ novel-coronavirus/en). Ces agents sont actuellement le lopinavirritonavir, le remdésivir et la chloroquine (ou hydroxychloroquine); à mesure que de nouvelles données se font jour, d'autres pourraient s'y ajouter. En l'absence de traitements efficaces éprouvés pour la COVID-19, démontrer qu'un médicament, quel qu'il soit, procure un bénéfice clinique net est une priorité de santé publique.

Plus de 500 essais cliniques sont déjà consignés au système d'enregistrement international mis sur pied par l'OMS à cet effet, et plusieurs recrutent activement des participants (https://apps. who.int/trialsearch), ce qui est à la fois impressionnant et préoccupant. Tant d'essais distincts pourraient comporter des risques de gaspillage et de double emploi et une concurrence malsaine pour recruter des participants dans un même bassin; certains pourraient avoir une puissance statistique insuffisante et entraîner le rejet prématuré de médicaments prometteurs ${ }^{6}$ ou leur accession trop hâtive au statut de norme thérapeutique. On évitera de tels risques en encourageant la collaboration entre les groupes de recherche du monde entier.

Nous croyons que le Canada doit veiller à la mise en place d'une infrastructure nécessaire pour soutenir le recrutement des patients atteints de la COVID-19 pour des essais sur des traitements afin que tous les médecins puissent vérifier si les patients qu'ils voient sont admissibles à la randomisation et, le cas échéant, leur offrir de participer à des essais cliniques réalisés en toute transparence et en maintenant le contact avec des partenaires ailleurs dans le monde. Cette stratégie rendrait les médecins confiants du fait qu'ils font le maximum pour leurs patients gravement malades tout en générant de nouvelles données. Les patients acceptent cette approche, comme en témoignent plusieurs études réalisées avant la pandémie actuelle $e^{7,8}$.

L'infrastructure canadienne en recherche clinique subit une mise à l'échelle rapide pour répondre aux impératifs scientifiques et éthiques, sur le modèle des essais qui ont été réalisés dans la foulée de l'épidémie d'Ebola9 ${ }^{9}$. Les Instituts de recherche en santé du Canada et leurs partenaires ont lancé en février 2020 une possibilité de financement pour une intervention de recherche rapide sur la COVID-19, qui a permis de rédiger des demandes, de faire des présentations, d'assurer la révision par des pairs et de rendre les décisions initiales concernant le financement en l'espace de 18 jours. Les instances de réglementation ont agi rapidement pour faciliter le démarrage des premiers essais canadiens sur la COVID-19.

Au Canada, le volumineux essai thérapeutique CATCO (Canadian Treatments for COVID-19) fera partie du projet SOLIDARITY de l'OMS, un protocole international visant à évaluer les meilleurs agents disponibles pour les patients hospitalisés. Le protocole de l'OMS a pour objectif d'inscrire des milliers de patients dans le monde afin d'obtenir rapidement la meilleure estimation possible de l'effet thérapeutique des agents prioritaires. Le Canada commencera par comparer le lopinavir-ritonavir aux soins de soutien optimisés et ajoutera de nouveaux agents selon les données générées. Étant donné qu'on ignore l'ampleur des effets, il est impossible de calculer précisément la taille de l'échantillon. L'approbation éthique de l'essai CATCO a été accordée en 5 jours ouvrables suivant la soumission du protocole en Ontario et en 10 jours en Colombie-Britannique. Au Québec et en Alberta les approbations sont à venir et Santé Canada a donné son approbation dans les 3 jours ouvrables suivant la demande initiale. Il faut se réjouir du soutien institutionnel sans précédent accordé à ces initiatives.

Pour sa part, REMAP-CAP (A Randomised, Embedded, Multifactorial, Adaptive Platform Trial for Community-Acquired Pneumonia) est un essai sur une plateforme adaptative établie avant la pandémie de COVID-19. Elle se penche sur diverses interventions chez les grands malades atteints de pneumonie ${ }^{10}$. Au Canada et dans 13 autres pays, l'essai inclut désormais des interventions spécifiques à la COVID-19 et des mesures de soutien, telles que macrolides, corticostéroïdes et interféron $\beta-1 a$, avec des antiviraux. CATCO et REMAP-CAP étudient leurs effets chez les grands malades, et le recrutement est coordonné entre les 2 essais. Les règles d'interruption et les paramètres principaux ont été modifiés et on prévoit l'ajout éventuel d'autres agents pour constituer plus rapidement une base de données de référence pendant cette pandémie.

Il est aussi crucial de générer des données probantes sur les mesures de prévention de la contamination, surtout chez les adultes âgés des centres de soins et les travailleurs de la santé dont il faut assurer la santé, tant physique que psychologique. L'étude CORIPREV LR (COVID-19 Ring-based Prevention Trial with Lopinavir/Ritonavir) évaluera l'efficacité du lopinavir-ritonavir en prophylaxie postexposition à prévenir une infection microbiologiquement avérée. Cette étude utilisera une approche adaptée d'un groupe d'essais randomisés réalisés durant l'épidémie d'Ebola en 2013-2016 en Afrique de l'Ouest ${ }^{11}$; les chercheurs identifieront les " cercles de contacts » des malades et les assigneront aléatoirement à la prophylaxie ou aux mesures préventives habituelles. Pour l'étude CORIPREV LR, 220 cercles seront assignés aléatoirement soit au lopinavir-ritonavir, soit à l'absence d'intervention, les participants des 2 groupes devant se soumettre à des auto-évaluations quotidiennes de leurs symptômes et à des tests de dépistage hebdomadaires. Rappelons que la vaccination des contacts a été fondamentale dans l'éradication de la variole ${ }^{12}$. L'infrastructure de l'essai CORIPREV LR pourra ensuite être adaptée afin d'évaluer rapidement l'efficacité des vaccins, lorsqu'ils seront disponibles.

Les défis posés aux ERC durant cette pandémie de COVID-19 relèvent du maintien d'un financement adéquat, d'une rapide mise à l'échelle et de la prévention de l'infection chez les chercheurs. De nouvelles stratégies pourraient être requises pour le consentement éclairé, entre autres, la mobilisation des ressources technologiques pour communiquer à distance. L'entrée des données pourrait être simplifiée en priorisant les éléments d'information les plus pertinents. Dans cette ligne de pensée, en collaboration avec des chercheurs principaux de l'ERC sur la prophylaxie postexposition à la COVID19 au Minnesota, le Canada permettra aux travailleurs de la santé et aux adultes vivant dans la communauté qui sont à risque élevé d'exposition ou qui présentent les premiers symptômes de la maladie de procéder eux-mêmes à un dépistage électronique, de recevoir le médicament qui leur aura été assigné 
(hydroxychloroquine c. placebo) par messagerie rapide et d'exercer un suivi par auto-évaluation. Les paramètres incluent la maladie symptomatique chez les individus initialement asymptomatiques et la maladie grave chez les individus initialement symptomatiques. Un ensemble de données regroupées permet de combiner les résultats canadiens aux résultats internationaux quasi en temps réel afin d'obtenir un échantillon dont la taille est estimée à 1500 patients par cohorte.

À mesure que la pandémie évolue, la tentation d'utiliser des médicaments non éprouvés sera très forte, mais en collaboration avec leurs collègues ailleurs dans le monde, les médecins canadiens doivent maintenir leur engagement à l'endroit d'une rigoureuse collecte de données scientifiques pour que nous puissions améliorer rapidement les résultats chez tous les patients atteints de la COVID-19 ici et ailleurs. Un registre informel des essais canadiens connus est accessible à l'adresse canada-covid.idtrials. com et sera mis à jour à mesure que de nouveaux renseignements seront disponibles.

\section{Références}

1. Wang M, Cao R, Zhang L, et al. Remdesivir and chloroquine effectively inhibit the recently emerged novel coronavirus (2019-nCoV) in vitro. Cell Res 2020;30:269-71.

2. Xu Z, Shi L, Wang Y, et al. Pathological findings of COVID-19 associated with acute respiratory distress syndrome. Lancet Respir Med le 18 février 2020 [Cyberpublication avant impression]. doi: 10.1016/S2213-2600(20)30076-X.
3. Herrera-Perez D, Haslam A, Crain T, et al. A comprehensive review of randomized clinical trials in three medical journals reveals 396 medical reversals. eLife 2019;8:e45183. doi: 10.7554/eLife.45183.

4. Dyer O. What did we learn from Tamiflu? BMJ 2020;368:m626.

5. Lai CC, Shih TP, Ko WC, et al. Severe acute respiratory syndrome coronavirus 2 (SARS-CoV-2) and coronavirus disease-2019 (COVID-19): the epidemic and the challenges. Int J Antimicrob Agents 2020;55:105924.

6. Cao B, Wang Y, Wen D, et al. A trial of lopinavir-ritonavir in adults hospitalized with severe COVID-19. N Engl J Med le 18 mars 2020 [Cyberpublication avant impression]. doi: 10.1056/NEJMoa2001282.

7. Gobat NH, Gal M, Butler CC, et al. Talking to the people that really matter about their participation in pandemic clinical research: a qualitative study in four European countries. Health Expect 2018;21:387-95.

8. Gobat N, Butler CC, Mollison J, et al. What the public think about participation in medical research during an influenza pandemic: an international crosssectional survey. Public Health 2019;177:80-94.

9. Mulangu S, Dodd LE, Davey RT Jr., et al.; PALM Consortium Study Team. A randomized, controlled trial of Ebola virus disease therapeutics. N Engl J Med 2019;381:2293-303.

10. REMAP-CAP: A Randomized, Embedded, Multi-factorial, Adaptive Platform Trial for Community-Acquired Pneumonia [page principale]. Accessible ici : www.remapcap.org (consulté le 3 mars 2020).

11. Henao-Restrepo AM, Camacho A, Longini IM, et al. Efficacy and effectiveness of an rVSV-vectored vaccine in preventing Ebola virus disease: final results from the Guinea ring vaccination, open-label, cluster-randomised trial (Ebola Ça Suffit!). Lancet 2017;389:505-18.

12. Kretzschmar M, van den Hof S, Wallinga J, et al. Ring vaccination and smallpox control. Emerg Infect Dis 2004;10:832-41.
Intérêts concurrents : Todd Lee reçoit un soutien à la rémunération du Fonds de recherche du Québec - Santé. L'établissement de Darrell Tan a reçu des subventions pour des travaux menés à l'initiative d'investigateurs ou un soutien non financier de Gilead, Viiv Healthcare et AbbVie. Darrell Tan est investigateur principal d'un site d'essais cliniques commandités par GlaxoSmithKline. Darrell Tan est titulaire d'une chaire de recherche du Canada de niveau 2. L'établissement de Srinivas Murthy a reçu un soutien non financier d'AbbVie.

Cet article n'a pas été révisé par des pairs.

Affiliations : Division d'infectiologie (Cheng, Lee), Département de médecine et Division de microbiologie médicale (Cheng), Département de méde- cine de laboratoire, Centre universitaire de santé McGill; Plateforme des essais cliniques (Cheng, Lee), Initiative interdisciplinaire en infection et immunité McGill, Montréal, Qué.; Division d'infectiologie (Tan) MAP Centre for Urban Health Solutions (Tan), Hôpital St. Michael; Département de médecine (Tan), Université de Toronto, Toronto, Ont.; Département de pédiatrie (Murthy), Université de la Colombie-Britannique, Vancouver, C.-B.

Collaborateurs : Tous les auteurs ont rédigé l'ébauche et révisé le manuscrit; ils ont donné leur approbation finale pour la version destinée à être publiée et assument l'entière responsabilité de tous les aspects du travail.

Adresse de correspondance : Srinivas Murthy, srinivas.murthy@cw.bc.ca 